\title{
CYCLIC REFINEMENTS OF THE DISCRETE HÖLDER'S INEQUALITY WITH APPLICATIONS
}

\author{
S. I. BUTT, L. HORVÁTH, AND J. PEČARIĆ \\ Received 10 March, 2020
}

\begin{abstract}
In this paper we obtain refinements of the discrete Hölder's and Minkowski's inequalities for finite and infinite sequences by using cyclic refinements of the discrete Jensen's inequality.

2010 Mathematics Subject Classification: 26A51;26D15

Keywords: discrete Jensen's inequality, discrete Hölder's inequality, discrete Minkowski’s inequality, convex function, cyclic refinement
\end{abstract}

\section{INTRODUCTION}

One of the most important inequalities concerning convex functions is the following

Theorem 1 (Discrete Jensen's inequality, [5]). Let $C$ be a convex subset of a real vector space $V$, and let $f: C \rightarrow \mathbb{R}$ be a convex function. If $p_{1}, \ldots, p_{n}$ are nonnegative numbers with $\sum_{i=1}^{n} p_{i}=1$, and $v_{1}, \ldots, v_{n} \in C$, then

$$
f\left(\sum_{i=1}^{n} p_{i} v_{i}\right) \leq \sum_{i=1}^{n} p_{i} f\left(v_{i}\right) .
$$

A number of attempts have been made to refine this inequality (see the book [5] and the references therein).

The following cyclic refinement of the discrete Jensen's inequality is a special case of Theorem 2.1 in the recent paper [6] (see also [1]). To give the result we need the following hypotheses:

\footnotetext{
The first author was supported by Higher Education Commission Pakistan under NRPU project 7906.

The research of the second author was supported by Hungarian National Research, Development and Innovation Office Grant No. KH130513, and Széchenyi 2020 under the EFOP-3.6.1-16-2016-00015.

The third author was supported by Ministry of Education and Science of the Russian Federation (the Agreement number No. 02.a03.21.0008.)
} 
$\left(\mathrm{H}_{1}\right)$ Let $2 \leq k \leq n$ be integers, let $\lambda_{1}, \ldots, \lambda_{k}$ represent a positive probability distribution, and let $p_{1}, \ldots, p_{n}$ be positive numbers with $P_{n}:=\sum_{i=1}^{n} p_{i}$.

$\left(\mathrm{H}_{2}\right)$ Let $I \subset \mathbb{R}$ be an interval, and $f: I \rightarrow \mathbb{R}$ be a convex function.

Theorem 2 (Theorem 2.1 in [6]). Assume $\left(H_{1}\right)$ and $\left(H_{2}\right)$. If $v_{1}, \ldots, v_{n} \in I$, then

$$
\begin{gathered}
f\left(\frac{1}{P_{n}} \sum_{i=1}^{n} p_{i} v_{i}\right) \leq C_{d i s}=C_{d i s}(f, \mathbf{v}, \mathbf{p}, \lambda) \\
:=\frac{1}{P_{n}} \sum_{i=1}^{n}\left(\sum_{j=0}^{k-1} \lambda_{j+1} p_{i+j}\right) f\left(\frac{\sum_{j=0}^{k-1} \lambda_{j+1} p_{i+j} v_{i+j}}{\sum_{j=0}^{k-1} \lambda_{j+1} p_{i+j}}\right) \leq \frac{1}{P_{n}} \sum_{i=1}^{n} p_{i} f\left(v_{i}\right)
\end{gathered}
$$

where $i+j$ means $i+j-n$ in case of $i+j>n$.

In this paper we first obtain cyclic refinements of the discrete Hölder's inequality by using the previous assertion. Then we give some refinements of the discrete Hölder's inequality for infinite sequences. There are a lot of papers dealing with similar refinements (see e.g. [2-4,7] and [8]). Our results fit well into the topic of refinements of inequalities corresponding to convex functions, and they give a new approach to have such refinements. Finally, we demonstrate the applicability of our results by means of some new cyclic refinements of the Minkowski's inequality.

\section{MAIN RESULTS}

Let $2 \leq k \leq n$, and let $i \in\{1, \ldots, n\}$ and $j \in\{0, \ldots, k-1\}$. In further parts of the paper $i+j$ always means $i+j-n$ in case of $i+j>n$.

In the first result we give cyclic refinements of the discrete Hölder's inequality.

Theorem 3. Let $2 \leq k \leq n$ be integers, and let $\lambda_{1}, \ldots, \lambda_{k}$ represent a positive probability distribution. Let $\left(w_{i}\right)_{i=1}^{n}$ be a sequence of positive numbers, $\left(x_{i}\right)_{i=1}^{n}$ be a sequence of nonnegative numbers, and let $p>0$ and $q \in \mathbb{R} \cup\{\infty\}$ be conjugate exponents that is $\frac{1}{p}+\frac{1}{q}=1$ (if $p=1$, we define its conjugate exponent to be $q=\infty$ ). $\left(a_{1}\right)$ If $\left(y_{i}\right)_{i=1}^{n}$ is a sequence of nonnegative numbers and $p>1$, then

$$
\begin{aligned}
\sum_{i=1}^{n} w_{i} x_{i} y_{i} & \leq \sum_{i=1}^{n}\left(\sum_{j=0}^{k-1} \lambda_{j+1} w_{i+j} x_{i+j}^{p}\right)^{\frac{1}{p}}\left(\sum_{j=0}^{k-1} \lambda_{j+1} w_{i+j} y_{i+j}^{q}\right)^{\frac{1}{q}} \\
& \leq\left(\sum_{i=1}^{n} w_{i} x_{i}^{p}\right)^{\frac{1}{p}}\left(\sum_{i=1}^{n} w_{i} y_{i}^{q}\right)^{\frac{1}{q}} .
\end{aligned}
$$


$\left(a_{2}\right)$ If $\left(y_{i}\right)_{i=1}^{n}$ is a sequence of nonnegative numbers, then

$$
\begin{aligned}
\sum_{i=1}^{n} w_{i} x_{i} y_{i} & \leq \sum_{i=1}^{n}\left(\sum_{j=0}^{k-1} \lambda_{j+1} w_{i+j} x_{i+j}\right) \cdot \max _{j \in\{0, \ldots, k-1\}} y_{i+j} \\
& \leq\left(\sum_{i=1}^{n} w_{i} x_{i}\right) \cdot \max _{i \in\{1, \ldots, n\}} y_{i} .
\end{aligned}
$$

$\left(a_{3}\right)$ If $\left(y_{i}\right)_{i=1}^{n}$ is a sequence of positive numbers and $0<p<1$, then the reverse inequalities hold in (2.1).

$\left(b_{1}\right)$ If $\left(y_{i}\right)_{i=1}^{n}$ is a sequence of nonnegative numbers and If $p>1$, then

$$
\begin{aligned}
\sum_{i=1}^{n} w_{i} x_{i} y_{i} & \leq\left(\sum_{i=1}^{n} w_{i} y_{i}^{q}\right)^{\frac{1}{q}}\left(\sum_{i=1}^{n}\left(\sum_{j=0}^{k-1} \lambda_{j+1} w_{i+j} y_{i+j}^{q}\right)^{1-p}\left(\sum_{j=0}^{k-1} \lambda_{j+1} w_{i+j} y_{i+j} x_{i+j}\right)^{p}\right)^{\frac{1}{p}} \\
& \leq\left(\sum_{i=1}^{n} w_{i} x_{i}^{p}\right)^{\frac{1}{p}}\left(\sum_{i=1}^{n} w_{i} y_{i}^{q}\right)^{\frac{1}{q}} .
\end{aligned}
$$

If $y_{i}=y_{i+1}=\ldots=y_{i+k-1}=0$ for some $i \in\{1, \ldots, n\}$, then

$$
\left(\sum_{j=0}^{k-1} \lambda_{j+1} w_{i+j} y_{i+j}^{q}\right)^{1-p}\left(\sum_{j=0}^{k-1} \lambda_{j+1} w_{i+j} y_{i+j} x_{i+j}\right)^{p}
$$

means

$$
\left(\sum_{j=0}^{k-1} \lambda_{j+1} w_{i+j}\right)^{1-p}\left(\sum_{j=0}^{k-1} \lambda_{j+1} w_{i+j} x_{i+j}\right)^{p} .
$$

$\left(b_{2}\right)$ If $\left(y_{i}\right)_{i=1}^{n}$ is a sequence of nonnegative numbers, then

$$
\begin{aligned}
\sum_{i=1}^{n} w_{i} x_{i} y_{i} & \leq \max _{i \in\{1, \ldots, n\}} y_{i} \cdot\left(\sum_{i=1}^{n} \frac{\sum_{j=0}^{k-1} \lambda_{j+1} w_{i+j} y_{i+j} x_{i+j}}{\max _{j \in\{0, \ldots, k-1\}} y_{i+j}}\right) \\
& \leq\left(\sum_{i=1}^{n} w_{i} x_{i}\right) \cdot \max _{i \in\{1, \ldots, n\}} y_{i} .
\end{aligned}
$$

If $y_{i}=y_{i+1}=\ldots=y_{i+k-1}=0$ for some $i \in\{1, \ldots, n\}$, then

$$
\frac{\sum_{j=0}^{k-1} \lambda_{j+1} w_{i+j} y_{i+j} x_{i+j}}{\max _{j \in\{0, \ldots, k-1\}} y_{i+j}}
$$


means

$$
\sum_{j=0}^{k-1} \lambda_{j+1} w_{i+j} x_{i+j}
$$

(b $b_{3}$ If $\left(y_{i}\right)_{i=1}^{n}$ is a sequence of positive numbers and $0<p<1$, then the reverse inequalities hold in (2.2).

Proof. Consider the power functions

$$
\left.f_{s}:\right] 0, \infty\left[\rightarrow \mathbb{R}, \quad f_{s}(x)=x^{s}, \quad s \neq 0,1 .\right.
$$

It is well known that $f_{s}$ is strictly convex for each $\left.s \in\right]-\infty, 0[\cup] 1, \infty[$, and strictly concave for each $s \in] 0,1\left[\right.$. By applying Theorem 2 to the function $f_{s}$ and to the positive numbers $v_{1}, \ldots, v_{n}$, we obtain that

$$
\left(\frac{1}{P_{n}} \sum_{i=1}^{n} p_{i} v_{i}\right)^{s} \leq \frac{1}{P_{n}} \sum_{i=1}^{n}\left(\sum_{j=0}^{k-1} \lambda_{j+1} p_{i+j}\right)^{1-s}\left(\sum_{j=0}^{k-1} \lambda_{j+1} p_{i+j} v_{i+j}\right)^{s} \leq \frac{1}{P_{n}} \sum_{i=1}^{n} p_{i} v_{i}^{s},
$$

if $s \in]-\infty, 0[\cup] 1, \infty[$, and the reverse inequality holds if $s \in] 0,1[$.

$\left(\mathrm{a}_{1}\right)$ Assume first that $x_{i}, y_{i}(i=1, \ldots, n)$ are positive numbers. By using the substitutions

$$
s=\frac{1}{p}, \quad p_{i}=w_{i} y_{i}^{q}, \quad v_{i}=x_{i}^{p} y_{i}^{-q}
$$

in (2.4), and by taking into account that $f_{1 / p}$ is concave, we obtain

$$
\begin{aligned}
\left(\frac{1}{\sum_{i=1}^{n} w_{i} y_{i}^{q}}\right)^{\frac{1}{p}}\left(\sum_{i=1}^{n} w_{i} x_{i}^{p}\right)^{\frac{1}{p}} & \geq \frac{1}{\sum_{i=1}^{n} w_{i} y_{i}^{q}} \cdot \sum_{i=1}^{n}\left(\sum_{j=0}^{k-1} \lambda_{j+1} w_{i+j} y_{i+j}^{q}\right) \cdot\left(\frac{\sum_{j=0}^{k-1} \lambda_{j+1} w_{i+j} x_{i+j}^{p}}{\sum_{j=0}^{k-1} \lambda_{j+1} w_{i+j} y_{i+j}^{q}}\right)^{\frac{1}{p}} \\
& \geq \frac{1}{\sum_{i=1}^{n} w_{i} y_{i}^{q}} \sum_{i=1}^{n} w_{i} x_{i} y_{i},
\end{aligned}
$$

and this is equivalent to (2.1).

In the general case let $\varepsilon>0$, apply the proved inequality to the positive sequences $\left(x_{i}+\varepsilon\right)_{i=1}^{n}$ and $\left(y_{i}+\varepsilon\right)_{i=1}^{n}$, and then take the limit as $\varepsilon \rightarrow 0+$.

$\left(\mathrm{a}_{2}\right)$ The inequality can be obtained by taking the limit as $q \rightarrow \infty$ in (2.1).

$\left(a_{3}\right)$ The reverse inequalities in $(2.1)$ can be proved exactly as in $\left(a_{1}\right)$ by considering that $f_{1 / p}$ is convex.

$\left(b_{1}\right)$ and $\left(b_{2}\right)$ can be proved similarly to $\left(a_{1}\right)$ and $\left(a_{2}\right)$ by using the substitutions

$$
s=p, \quad p_{i}=w_{i} y_{i}^{q}, \quad v_{i}=x_{i} y_{i}^{1-q}
$$

in (2.4).

$\left(b_{3}\right)$ The reverse inequalities in (2.2) can be proved exactly as in $\left(b_{1}\right)$ by considering that the function $f_{p}$ is concave. 
The proof is complete.

The following new inequalities follow from the previous result.

Corollary 1. Let $2 \leq k \leq n$ be integers, and let $\lambda_{1}, \ldots, \lambda_{k}$ represent a positive probability distribution. Let $\left(w_{i}\right)_{i=1}^{n}$ and $\left(y_{i}\right)_{i=1}^{n}$ be sequences of positive numbers, $\left(x_{i}\right)_{i=1}^{n}$ be a sequence of nonnegative numbers, and let $p>0$ and $q \in \mathbb{R} \cup\{\infty\}$ be conjugate exponents.

(a) If $p>1$, then

$$
\sum_{i=1}^{n}\left(\sum_{j=0}^{k-1} \lambda_{j+1} w_{i+j} y_{i+j}^{q}\right)^{1-p}\left(\sum_{j=0}^{k-1} \lambda_{j+1} w_{i+j} y_{i+j} x_{i+j}\right)^{p} \leq \sum_{i=1}^{n} w_{i} x_{i}^{p},
$$

while if $0<p<1$, then the reverse inequality holds.

(b) If $p=1$, then

$$
\sum_{i=1}^{n} \frac{\sum_{j=0}^{k-1} \lambda_{j+1} w_{i+j} y_{i+j} x_{i+j}}{\max _{j \in\{0, \ldots, k-1\}} y_{i+j}} \leq \sum_{i=1}^{n} w_{i} x_{i} .
$$

Proof. Theorem $3\left(\mathrm{~b}_{1}\right),\left(\mathrm{b}_{2}\right)$ and $\left(\mathrm{b}_{3}\right)$ imply these inequalities.

As an immediate consequence of Theorem 3 we obtain cyclic refinements of the Cauchy-Schwarz-Bunyakovszkij inequality.

Corollary 2. Let $2 \leq k \leq n$ be integers, let $\lambda_{1}, \ldots, \lambda_{k}$ represent a positive probability distribution, and let $\left(x_{i}\right)_{i=1}^{n}$ and $\left(y_{i}\right)_{i=1}^{n}$ be sequences of real numbers. Then (a)

$$
\begin{aligned}
\left|\sum_{i=1}^{n} w_{i} x_{i} y_{i}\right| & \leq \sum_{i=1}^{n}\left(\sum_{j=0}^{k-1} \lambda_{j+1} w_{i+j} x_{i+j}^{2}\right)^{\frac{1}{2}}\left(\sum_{j=0}^{k-1} \lambda_{j+1} w_{i+j} y_{i+j}^{2}\right)^{\frac{1}{2}} \\
& \leq\left(\sum_{i=1}^{n} w_{i} x_{i}^{2}\right)^{\frac{1}{2}}\left(\sum_{i=1}^{n} w_{i} y_{i}^{2}\right)^{\frac{1}{2}} .
\end{aligned}
$$

(b)

$$
\begin{aligned}
\left|\sum_{i=1}^{n} w_{i} x_{i} y_{i}\right| & \leq\left(\sum_{i=1}^{n} w_{i} y_{i}^{2}\right)^{\frac{1}{2}}\left(\sum_{i=1}^{n} \frac{\left(\sum_{j=0}^{k-1} \lambda_{j+1} w_{i+j}\left|y_{i+j}\right|\left|x_{i+j}\right|\right)^{2}}{\sum_{j=0}^{k-1} \lambda_{j+1} w_{i+j} y_{i+j}^{2}}\right)^{\frac{1}{2}} \\
& \leq\left(\sum_{i=1}^{n} w_{i} x_{i}^{2}\right)^{\frac{1}{2}}\left(\sum_{i=1}^{n} w_{i} y_{i}^{2}\right)^{\frac{1}{2}} .
\end{aligned}
$$


If $y_{i}=y_{i+1}=\ldots=y_{i+k-1}=0$ for some $i \in\{1, \ldots, n\}$, then

$$
\frac{\left(\sum_{j=0}^{k-1} \lambda_{j+1} w_{i+j}\left|y_{i+j}\right|\left|x_{i+j}\right|\right)^{2}}{\sum_{j=0}^{k-1} \lambda_{j+1} w_{i+j} y_{i+j}^{2}}
$$

means

$$
\frac{\left(\sum_{j=0}^{k-1} \lambda_{j+1} w_{i+j}\left|x_{i+j}\right|\right)^{2}}{\sum_{j=0}^{k-1} \lambda_{j+1} w_{i+j}} .
$$

Proof. Choose $p=2$ in Theorem $3\left(\mathrm{a}_{1}\right)$ and $\left(\mathrm{b}_{1}\right)$.

By Theorem 3, some refinements of the Hölder's inequality for infinite sequences can be obtained. We have to be very careful, because a change in $n$ causes a corresponding change in $i+j$, that is $i+j$ depends on $n$. We illustrate this by the generalization of inequality (2.1).

Theorem 4. Let $k \geq 2$ be an integer, and let $\lambda_{1}, \ldots, \lambda_{k}$ represent a positive probability distribution. Let $\left(w_{i}\right)_{i=1}^{\infty}$ be a sequence of positive numbers, and let $\left(x_{i}\right)_{i=1}^{\infty}$ and $\left(y_{i}\right)_{i=1}^{\infty}$ be sequences of nonnegative numbers such that

$$
\sum_{i=1}^{\infty} w_{i} x_{i}^{p}<\infty, \quad \sum_{i=1}^{\infty} w_{i} y_{i}^{q}<\infty,
$$

where $p>0$ and $q \in \mathbb{R} \cup\{\infty\}$ are conjugate exponents.

(a) If $p>1$, then

$$
\begin{aligned}
\sum_{i=1}^{\infty} w_{i} x_{i} y_{i} \leq & \sum_{i=1}^{\infty}\left(\sum_{l=i}^{i+k-1} \lambda_{l-i+1} w_{l} x_{l}^{p}\right)^{\frac{1}{p}}\left(\sum_{l=i}^{i+k-1} \lambda_{l-i+1} w_{l} y_{l}^{q}\right)^{\frac{1}{q}} \\
& +\sum_{m=1}^{k-1}\left(\sum_{j=k-m}^{k-1} \lambda_{j+1} w_{m-k+1+j} x_{m-k+1+j}^{p}\right)^{\frac{1}{p}}\left(\sum_{j=k-m}^{k-1} \lambda_{j+1} w_{m-k+1+j} y_{m-k+1+j}^{q}\right)^{\frac{1}{q}} \\
\leq & \left(\sum_{i=1}^{\infty} w_{i} x_{i}^{p}\right)^{\frac{1}{p}}\left(\sum_{i=1}^{\infty} w_{i} y_{i}^{q}\right)^{\frac{1}{q}}
\end{aligned}
$$

(b) If $y_{i}>0(i=1,2, \ldots)$ and $0<p<1$, then the reverse inequalities hold in (2.5).

Proof. (a) It follows from Theorem $3\left(\mathrm{a}_{1}\right)$ that for each fixed $n \geq k$

$$
\sum_{i=1}^{n} w_{i} x_{i} y_{i} \leq \sum_{i=1}^{n}\left(\sum_{j=0}^{k-1} \lambda_{j+1} w_{i+j} x_{i+j}^{p}\right)^{\frac{1}{p}}\left(\sum_{j=0}^{k-1} \lambda_{j+1} w_{i+j} y_{i+j}^{q}\right)^{\frac{1}{q}}
$$




$$
\begin{aligned}
= & \sum_{i=1}^{n-k+1}\left(\sum_{j=0}^{k-1} \lambda_{j+1} w_{i+j} x_{i+j}^{p}\right)^{\frac{1}{p}}\left(\sum_{j=0}^{k-1} \lambda_{j+1} w_{i+j} y_{i+j}^{q}\right)^{\frac{1}{q}} \\
& +\sum_{i=n-k+2}^{n}\left(\sum_{j=0}^{n-i} \lambda_{j+1} w_{i+j} x_{i+j}^{p}+\sum_{j=n-i+1}^{k-1} \lambda_{j+1} w_{i+j} x_{i+j}^{p}\right)^{\frac{1}{p}} \\
& \cdot\left(\sum_{j=0}^{n-i} \lambda_{j+1} w_{i+j} y_{i+j}^{q}+\sum_{j=n-i+1}^{k-1} \lambda_{j+1} w_{i+j} y_{i+j}^{q}\right)^{\frac{1}{q}} \\
= & \sum_{i=1}^{n-k+1}\left(\sum_{l=i}^{i+k-1} \lambda_{l-i+1} w_{l} x_{l}^{p}\right)^{\frac{1}{p}}\left(\sum_{l=i}^{i+k-1} \lambda_{l-i+1} w_{l} y_{l}^{q}\right)^{\frac{1}{q}} \\
& +\sum_{m=1}^{k-1}\left(\sum_{j=0}^{k-m-1} \lambda_{j+1} w_{m+n-k+1+j} x_{m+n-k+1+j}^{p}+\sum_{j=k-m}^{k-1} \lambda_{j+1} w_{m-k+1+j} x_{m-k+1+j}^{p}\right)^{\frac{1}{p}} \\
& \cdot\left(\sum_{j=0}^{k-m-1} \lambda_{j+1} w_{m+n-k+1+j} y_{m+n-k+1+j}^{q}+\sum_{j=k-m}^{k-1} \lambda_{j+1} w_{m-k+1+j} y_{m-k+1+j}^{q}\right)^{\frac{1}{q}} .
\end{aligned}
$$

Now, as $n$ tends to infinity, we obtain the result.

(b) We can prove as in (a) by using Theorem $3\left(a_{3}\right)$.

The proof is complete.

\section{ApPliCATiOns}

In the first result a cyclic refinement of the Minkowski's inequality is given.

Theorem 5. Let $2 \leq k \leq n$ be integers, let $\lambda_{1}, \ldots, \lambda_{k}$ represent a positive probability distribution, and let $\left(x_{i}\right)_{i=1}^{n}$ and $\left(y_{i}\right)_{i=1}^{n}$ be sequences of real numbers. If $p>1$ and $q>1$ are conjugate exponents, and there exists $i \in\{1, \ldots, n\}$ such that $x_{i}+y_{i} \neq 0$, then

$$
\begin{aligned}
\left(\sum_{i=1}^{n} w_{i}\left|x_{i}+y_{i}\right|^{p}\right)^{\frac{1}{p}} \leq & \frac{1}{\left(\sum_{i=1}^{n} w_{i}\left|x_{i}+y_{i}\right|^{p}\right)^{\frac{1}{q}}} \sum_{i=1}^{n}\left(\sum_{j=0}^{k-1} \lambda_{j+1} w_{i+j}\left|x_{i+j}+y_{i+j}\right|^{p}\right)^{\frac{1}{q}} \\
& \cdot\left(\left(\sum_{j=0}^{k-1} \lambda_{i+j} w_{i+j}\left|x_{i+j}\right|^{p}\right)^{\frac{1}{p}}+\left(\sum_{j=0}^{k-1} \lambda_{i+j} w_{i+j}\left|y_{i+j}\right|^{p}\right)^{\frac{1}{p}}\right) \\
\leq & \left(\sum_{i=1}^{n} w_{i}\left|x_{i}\right|^{p}\right)^{\frac{1}{p}}+\left(\sum_{i=1}^{n} w_{i}\left|y_{i}\right|^{p}\right)^{\frac{1}{p}} .
\end{aligned}
$$


Proof. A similar argument as in the proof of the classical Minkowski's inequality can be applied.

By Theorem $3\left(\mathrm{a}_{1}\right)$,

$$
\begin{aligned}
& \sum_{i=1}^{n} w_{i}\left|x_{i}+y_{i}\right|^{p} \leq \sum_{i=1}^{n} w_{i}\left|x_{i}\right|\left|x_{i}+y_{i}\right|^{p-1}+\sum_{i=1}^{n} w_{i}\left|y_{i}\right|\left|x_{i}+y_{i}\right|^{p-1} \\
& \leq \sum_{i=1}^{n}\left(\sum_{j=0}^{k-1} \lambda_{i+j} w_{i+j}\left|x_{i+j}\right|^{p}\right)^{\frac{1}{p}}\left(\sum_{j=0}^{k-1} \lambda_{j+1} w_{i+j}\left|x_{i+j}+y_{i+j}\right|^{p}\right)^{\frac{1}{q}} \\
& \quad+\sum_{i=1}^{n}\left(\sum_{j=0}^{k-1} \lambda_{i+j} w_{i+j}\left|y_{i+j}\right|^{p}\right)^{\frac{1}{p}}\left(\sum_{j=0}^{k-1} \lambda_{j+1} w_{i+j}\left|x_{i+j}+y_{i+j}\right|^{p}\right)^{\frac{1}{q}} \\
& \leq\left(\sum_{i=1}^{n} w_{i}\left|x_{i}\right|^{p}\right)^{\frac{1}{p}} \cdot\left(\sum_{i=1}^{n} w_{i}\left|x_{i}+y_{i}\right|^{p}\right)^{\frac{1}{q}}+\left(\sum_{i=1}^{n} w_{i}\left|y_{i}\right|^{p}\right)^{\frac{1}{p}} \cdot\left(\sum_{i=1}^{n} w_{i}\left|x_{i}+y_{i}\right|^{p}\right)^{\frac{1}{q}},
\end{aligned}
$$

and this implies the result.

The proof is complete.

Remark 1. By using Theorem $3\left(\mathrm{~b}_{1}\right)$, we can obtain another cyclic refinement of the Minkowski's inequality.

Related to Theorem 4 we have the following cyclic refinement of the Minkowski's inequality for infinite sums. For simplicity and transparency we consider only the case $k=2$.

Theorem 6. Let $\lambda_{1}$ and $\lambda_{2}$ be positive numbers with $\lambda_{1}+\lambda_{2}=1$. Let $\left(w_{i}\right)_{i=1}^{\infty}$ be a sequence of positive numbers, and let $\left(x_{i}\right)_{i=1}^{\infty}$ and $\left(y_{i}\right)_{i=1}^{\infty}$ be sequences of real numbers such that

$$
\sum_{i=1}^{\infty} w_{i}\left|x_{i}\right|^{p}<\infty, \quad \sum_{i=1}^{\infty} w_{i}\left|y_{i}\right|^{q}<\infty
$$

where $p>1$ and $q>1$ are conjugate exponents. If there exists $i \in\{1,2, \ldots\}$ such that $x_{i}+y_{i} \neq 0$, then

$$
\begin{aligned}
\left(\sum_{i=1}^{\infty} w_{i}\left|x_{i}+y_{i}\right|^{p}\right)^{\frac{1}{p}} \leq & \frac{1}{\left(\sum_{i=1}^{\infty} w_{i}\left|x_{i}+y_{i}\right|^{p}\right)^{\frac{1}{q}}} \cdot\left(\sum_{i=1}^{\infty}\left(\lambda_{1} w_{i}\left|x_{i}+y_{i}\right|^{p}+\lambda_{2} w_{i+1}\left|x_{i+1}+y_{i+1}\right|^{p}\right)^{\frac{1}{q}}\right. \\
& \left(\left(\lambda_{1} w_{i}\left|x_{i}\right|^{p}+\lambda_{2} w_{i+1}\left|x_{i+1}\right|^{p}\right)^{\frac{1}{p}}+\left(\lambda_{1} w_{i}\left|y_{i}\right|^{p}+\lambda_{2} w_{i+1}\left|y_{i+1}\right|^{p}\right)^{\frac{1}{p}}\right) \\
& \left.+\left(\lambda_{2} w_{1}\left|x_{1}+y_{1}\right|^{p}\right)^{\frac{1}{q}}\left(\left(\lambda_{2} w_{1}\left|x_{1}\right|^{p}\right)^{\frac{1}{p}}+\left(\lambda_{2} w_{1}\left|y_{1}\right|^{p}\right)^{\frac{1}{p}}\right)\right) \\
\leq & \left(\sum_{i=1}^{\infty} w_{i}\left|x_{i}\right|^{p}\right)^{\frac{1}{p}}+\left(\sum_{i=1}^{\infty} w_{i}\left|y_{i}\right|^{p}\right)^{\frac{1}{p}}
\end{aligned}
$$


Proof. We can follow the proof of Theorem 5, by using Theorem 4 instead of Theorem $3\left(\mathrm{a}_{1}\right)$.

\section{REFERENCES}

[1] I. Brnetić, K. A. Khan, and J. Pečarić, "Refinement of Jensen's inequality with applications to cyclic mixed symmetric means and Cauchy means," J. Math. Inequal, vol. 9, no. 4, pp. 1309-1321, 2015, doi: doi:10.7153/jmi-09-100.

[2] L. Ciurdariu, "Some refinements of Hölder's inequalities via isotonic linear functionals," Journal of Science and Arts, vol. 14, no. 3, p. 221, 2014.

[3] G. Horváth, "Refinements of Hölder's inequality," Acta Mathematica Hungarica, vol. 144, no. 1, pp. 110-118, 2014, doi: doi:10.1007/s10474-014-0423-3.

[4] L. Horváth, K. A. Khan, and J. Pečarić, "Refinements of Hölder and Minkowski inequalities with weights," Proceedings of A. Razmadze Mathematical Institute, vol. 158, pp. 33-56, 2012.

[5] L. Horváth, K. A. Khan, and J. Pečarić, Combinatorial Improvements of Jensen's Inequality. Zagreb: ELE-MATH, Monographs in Inequalities 8., 2014.

[6] L. Horváth, K. A. Khan, and J. Pečarić, "Cyclic refinements of the discrete and integral form of Jensen's inequality with applications," Analysis, vol. 36, no. 4, pp. 253-262, 2016, doi: 10.1515/anly-2015-0022.

[7] H. Qiang and Z. Hu, "Generalizations of Hölder's and some related inequalities," Computers \& Mathematics with Applications, vol. 61, no. 2, pp. 392-396, 2011, doi: doi.org/10.1016/j.camwa.2010.11.015.

[8] J. Tian, “A new refinement of generalized Hölder's inequality and its application,” Journal of Function Spaces, vol. 2013, 2013, doi: doi.org/10.1155/2013/686404.

Authors' addresses

S. I. Butt

COMSATS, University Islamabad, Lahore Campus, Lahore, Pakistan

E-mail address: saadihsanbutt@gmail.com

\section{Horváth}

University of Pannonia, Department of Mathematics, Egyetem u. 10. 8200 Veszprém, Hungary

E-mail address: lhorvathealmos.uni-pannon.hu

J. Pečarić

RUDN University Moscow, Miklukho-Maklaya str. 6, 117198, Russia

E-mail address: pecaric@mahazu.hazu.hr 